\title{
Protective role of isoflurane pretreatment in rats with focal cerebral ischemia and the underlying molecular mechanism
}

\author{
ZHIBIN XIAO ${ }^{1,2 *}$, PENGCHENG REN $^{1 *}$, YANG CHAO $^{3}$, QIANYUN WANG $^{4}$, JIANKE KUAI $^{1}$, \\ MIAOMIAO LV ${ }^{2}$, LEI CHEN ${ }^{5}$, CHANGJUN GAO ${ }^{1}$ and XUDE SUN ${ }^{1}$ \\ ${ }^{1}$ Department of Anesthesiology, Tangdou Hospital, The Fourth Military Medical University, \\ Xi'an, Shaanxi 710032; Departments of ${ }^{2}$ Anesthesiology, ${ }^{3}$ Stomatology and ${ }^{4}$ Medicine, \\ The 323 Hospital of People's Liberation Army, Xi'an, Shaanxi 710054; ${ }^{5}$ Department of Obstetrics and Gynecology, \\ The Navy General Hospital of People's Liberation Army, Beijing 100059, P.R. China
}

Received March 15, 2014; Accepted November 3, 2014

DOI: $10.3892 / \mathrm{mmr} .2015 .3408$

\begin{abstract}
Inflammation and immunity are important in the pathogenesis of cerebral ischemia. Toll-like receptor 4 (TLR4) is involved in the inflammatory responses of injured brain tissues. Emerging studies have focused on the effect of isoflurane (ISO) pretreatment on cerebral ischemia, however, the association between ISO pretreatment and TLR4 during cerebral ischemia remains to be elucidated. In the present study, the protective role of ISO pretreatment in rats with focal cerebral ischemia reperfusion was investigated and the molecular mechanism was discussed. Using a middle cerebral artery occlusion (MCAO) model, triphenyltetrazolium chloride staining was utilized to measure the infarct volume and brain edema and immunofluorescence staining was used to detect the MCAO-induced TLR4 expression and localization. Western blot analyses were conducted to quantify the protein expression levels of TLR4, myeloid differentiation primary response 88 (MyD88) and nuclear factor $(\mathrm{NF})-\kappa \mathrm{B}$ in ischemic brain tissue at different time points. The results demonstrated that, following ISO pretreatment, the neurological deficits, brain edema and cerebral infarct size caused by ischemia/reperfusion were attenuated. The astrocyte and microglial activation in the brain tissue was decreased. In addition, the expression levels of TLR4, MyD88 and NF-кB were decreased. The
\end{abstract}

Correspondence to: Dr Xude Sun, Department of Anesthesiology, Tangdou Hospital, The Fourth Military Medical University, 1 Xinsi Road, Baqiao, Xi'an, Shaanxi 710032, P.R. China

E-mail: xudesun@yeah.net

Dr Lei Chen, Department of Obstetrics and Gynecology, The Navy General Hospital of People's Liberation Army, 6 Fucheng Road, Haidian, Beijing 100059, P.R. China.

E mail: chenleis@mail.tsinghua.edu.cn

*Contributed equally

Key words: isoflurane, protective, cerebral ischemia present study indicated that ISO pretreatment may protect the brain from ischemic damage by downregulating the expression levels of TLR4, MyD88 and NF- $\mathrm{BB}$.

\section{Introduction}

Inflammation is important in the pathology of cerebral ischemic injury. Cerebral ischemia induces a marked inflammatory response characterized by the activation and release of cytokines, chemokines, adhesion molecules and endotoxins, which exacerbate tissue damage (1). Toll-like receptors (TLRs) have important functions in cerebral ischemia/reperfusion injury (2). TLRs and their ligands provide an important molecular interface between microbes and immunity and experimental cerebral ischemia upregulates TLR4 expression $(3,4)$. In addition, the brain damage and neurological deficits produced by cerebral ischemia significantly decrease in TLR4-deficient mice compared with wild-type mice (5). Volatile anesthetic pretreatment significantly protects against cerebral ischemia through several mechanisms $(6,7)$. Isoflurane (ISO) pretreatment inhibits excitotoxicity and reduces glutamate release from anoxic brain slices in vitro (8). ISO also inhibits postsynaptic glutamate receptor-mediated responses in neocortical and hippocampal cells (9-11) and rat brain slices (12). However, the exact mechanisms underlying this protective effect remain to be elucidated. Considering the function of the TLR4-mediated myeloid differentiation primary response 88 (MyD88)-dependent signaling pathway, the present study hypothesized that ISO pretreatment is associated with the TLR4 signaling pathway during cerebral ischemia/reperfusion damage. The association between the TLR4-induced inflammatory response and ISO pretreatment has attracted considerable interest in developing anti-inflammatory therapies to combat ischemia-induced damage.

Astrocytes are the most abundant type of glial cell within the brain. Although microglial cells are the first inflammatory cells to respond to cerebral ischemia, the responses of astrocytes to pro-inflammatory signals may also be relevant to brain injury (13-15). However, the association between inflammatory glial cells and TLR4 has not been fully elucidated. The effects of ISO pretreatment on TLR4 expression in astrocytes 
and microglia were investigated to increase understanding of the association between glial cells and TLR4 signaling pathways during inflammation. The anti-inflammatory effects associated with the TLR4-mediated MyD88 activation pathway in the cerebral ischemic brain were examined and the downstream molecules present in rats with cerebral ischemia were further investigated.

\section{Materials and methods}

Animal surgical procedures. ISO was purchased from Abbott Laboratories (North Chicago, IL, USA) and was stored in the dark. Pretreatment with $2 \%$ ISO for 60 min was deemed sufficient to induce ischemic tolerance $(16,17)$. Male Sprague-Dawley rats weighing between 250 and $270 \mathrm{~g}$ were provided by the Experimental Animal Center of the Fourth Military Medical University (Xi'an, China). The present study strictly followed the recommendations of the Guide for the Care and Use of Laboratory Animals of the National Institutes of Health (Bethesda, MD, USA). The animal use protocol was reviewed and approved by the Institutional Animal Care and Use Committee of the Fourth Military Medical University. Cerebral ischemia was induced via middle cerebral artery occlusion (MCAO) using the technique by Longa et al $(18,19)$. The right common carotid artery and the right external carotid artery (ECA) were exposed through a ventral midline neck incision and ligated proximally. A 3-0 nylon monofilament with a silicone-coated tip was inserted from the distal end of the isolated ECA and gently introduced to the internal carotid artery (ICA). The microfilament was then advanced into the ICA $\sim 18-20 \mathrm{~mm}$ distal to the carotid bifurcation until mild resistance was felt. The neck incision was closed with sutures and was left in place for $120 \mathrm{~min}$ to induce ischemia prior to reperfusion. The rectal temperatures were monitored and maintained between 37.0 and $37.5^{\circ} \mathrm{C}$ during the procedure.

Experimental procedures. The rats were randomly assigned into naive, sham- $\mathrm{O}_{2}$, sham-ISO, MCAO- $\mathrm{O}_{2}$ and MCAO-ISO groups to detect the effects of ISO pretreatment on the infarct volume, water content, immunofluorescence staining and protein expression of TLR4, MyD88 and nuclear factor $(\mathrm{NF})-\kappa \mathrm{B}$ in cerebral ischemia. The $\mathrm{MCAO}-\mathrm{O}_{2}$ group received $60 \% \mathrm{O}_{2} / 40 \% \mathrm{~N}_{2}$ at $60 \mathrm{~min}$ and $24 \mathrm{~h}$ after the last pretreatment, followed by the induction of focal cerebral ischemia. The MCAO-ISO group received 2\% ISO in $\mathrm{O}_{2}$ at $60 \mathrm{~min}$ and $24 \mathrm{~h}$ after the last pretreatment, followed by the induction of focal cerebral ischemia. The sham- $\mathrm{O}_{2}$ group received $\left(60 \% \mathrm{O}_{2} / 40 \%\right.$ $\mathrm{N}_{2}$ ) at $60 \mathrm{~min}$ and $24 \mathrm{~h}$ after the last pretreatment, whereas the sham-ISO group received $2 \%$ ISO in $\mathrm{O}_{2}$ at $60 \mathrm{~min}$ and $24 \mathrm{~h}$ after the last pretreatment. The right common carotid artery and the right ECA were subsequently exposed without the insertion of the monofilament into the artery. The temperature was maintained at $\sim 25^{\circ} \mathrm{C}$ during and following surgery. The animals were exposed to incandescent lamps to maintain their rectal temperatures at $37.0 \pm 0.5^{\circ} \mathrm{C}$ until pallanesthesia.

Neurological assessment. At $24 \mathrm{~h}$ after reperfusion, the rats in each group $(n=12)$ were neurologically assessed by an examiner in a blinded manner. The deficits were scored using a modified scoring system based on the system developed by
Longa et al (19): 0, no deficits; 1, difficulty in fully extending the contralateral forelimb; 2 , unable to extend the contralateral forelimb; 3 , mild circling to the contralateral side; 4 , severe circling and 5, falling to the contralateral side.

Measurement of infarct volume. The rats in each group $(n=12)$ were anesthetized with intraperitoneal sodium pentobarbital $(50 \mathrm{mg} / \mathrm{kg}) 24 \mathrm{~h}$ after ischemia. The brains were removed quickly and subsequently cut into a total of seven $2 \mathrm{~mm}$ coronal sections. The sections were immersed for $30 \mathrm{~min}$ in $2 \%$ triphenyltetrazolium chloride at $37^{\circ} \mathrm{C}$ and subsequently fixed with $4 \%$ paraformaldehyde. At $24 \mathrm{~h}$ after fixation, images were captured of the brain slices with a digital camera (Sony A350; Sony Corporation, Tokyo, Japan) connected to a personal computer.

Assessment of brain edema (brain water content). Brain water content was measured using the dry-wet weight technique (20). A total of 12 rats in each group were sacrificed and their brain tissues were quickly harvested. The $2 \mathrm{~mm}$-thick frontal pole was removed. The $2 \mathrm{~mm}$-thick brain tissue posterior to the frontal pole was selected to measure water content. Following determination of the wet weight (WW) using an electronic balance, the brain tissue was placed in an oven at constant temperature $\left(100 \pm 5^{\circ} \mathrm{C}\right)$ for $24 \mathrm{~h}$. The dry weight (DW) was subsequently measured. Water content was calculated based on the following formula: (WW - DW) / WW x 100.

Immunohistochemistry. Immunofluorescence photomicrographs of the lesion-associated cortex were obtained following the MCAO treatment. After $24 \mathrm{~h}$, the rats were anaesthetized using $6.0 \%$ isoflurane and their brains were removed quickly and immersed in $4 \%$ paraformaldehyde/0.1 M phosphate-buffered saline (PBS) for $2 \mathrm{~h}$ at $4^{\circ} \mathrm{C}$. The cryostat sections $(25 \mu \mathrm{m})$ in the first dish were rinsed three times (10 min each) in $0.01 \mathrm{M}$ PBS, pH 7.3 and subsequently blocked with $2 \%$ goat serum in 0.01 M PBS containing $0.3 \%$ Triton X-100 for $1 \mathrm{~h}$ at room temperature. The samples were then subjected to immunofluorescence staining.

To evaluate colocalization, a double immunohistochemical analysis was performed, in which sections were incubated for $48 \mathrm{~h}$ at $4^{\circ} \mathrm{C}$ with the primary antibodies TLR4 goat polyclonal antibodies (1:200; Abcam, Cambridge, UK) with mouse anti-cd11b clone OX-42 (1:300; Abcam) or mouse anti-glial fibrillary acidic protein (GFAP; 1:5,000; Chemicon, Temecula, CA, USA). The sections were washed three times in $0.01 \mathrm{M}$ PBS (10 min each) and then incubated for $4 \mathrm{~h}$ at room temperature with the secondary antibodies: Fluorescein isothiocyanate-conjugated horse anti-mouse immunoglobulin G (1:200; Vector, Burlingame, CA, USA) and Alexa 594-conjugated donkey anti-rabbit IgG (1:800; Molecular Probes, Rockford, IL, USA). For TLR4/GFAP and TLR4/OX-42 double immunofluorescence, the sections were incubated with a mixture of the two primary antibodies, followed by a mixture of the two respective secondary antibodies. The staining specificities were assessed on the sections in the second dish by omitting the specific primary antibodies. No immunoreactive (IR) products were identified on the sections (data not shown). Confocal images were obtained using a confocal laser microscope (FV1000; Olympus, 
Tokyo, Japan) and digital images were captured with the Fluoview 1000 microscope (Olympus).

Western blot analysis. The protein expression levels of MyD88, NF-кB and TLR4 were detected using western blot analysis. The tissue samples obtained from the right ischemic MCA were subjected to $120 \mathrm{~min}$ of MCA occlusion, with reperfusion after 24,48 and $72 \mathrm{~h}$. The tissues were subsequently lysed with radio-immunoprecipitation assay lysis buffer, homogenized and then centrifuged at 12,000 rpm for $20 \mathrm{~min}$ at $4^{\circ} \mathrm{C}$. The protein content of the supernatants were quantified using a bicinchoninic acid protein assay reagent kit (Beyotime Institute of Biotechnology, Shanghai, China). The remaining supernatants were boiled in sodium dodecyl sulfate (SDS) sample buffer for $5 \mathrm{~min}$. Equal quantities of the protein were run on SDS/polyacrylamide gel electrophoresis and electrophoretically transferred onto polyvinylidene fluoride membranes. Following blocking, the blots were immersed overnight in TLR4 goat polyclonal antibodies (1:200; Abcam), MyD88 rabbit polyclonal antibodies (1:200; Abcam) or NF $\kappa \mathrm{B}$ rabbit polyclonal antibodies $\left(1: 400\right.$; Abcam) at $4^{\circ} \mathrm{C}$. The membranes were rinsed and further incubated for $1 \mathrm{~h}$ with horseradish peroxidase-conjugated secondary antibodies (1:1,000; Vector). The bound antibodies were exposed to an Amersham Hyperfilm ${ }^{\mathrm{TM}}$ ECL (GE Healthcare, Amersham, UK). The relative densities of the bands were analyzed and were corrected using the values determined with anti-rat $\beta$-actin, which was used as the internal control. The densities of the protein blots were analyzed using Labworks Software Gel-Pro analyzer 4.0 (Ultra-Violet Products, Cambridge, UK). The densities of TLR4, MyD88, NF- $\kappa$ B and $\beta$-actin IR bands were quantified with background subtraction. Squares of identical sizes were drawn surrounding each band to measure density and the background near that band was subtracted. The $\beta$-actin levels were used as loading controls as they did not alter significantly following inflammation and nerve injury (21). The expression levels of TLR4, MyD88 and NF- $\mathrm{B}$ were normalized against the $\beta$-actin levels and were expressed as fold change relative to the control.

Quantification and statistical analysis. All the data were collected and analyzed by researchers in a blinded manner. A total of five nonadjacent sections $(25 \mu \mathrm{m})$ from the lesion-associated cortex sections were randomly selected. In each group, 12 rats were used for statistical analysis. The images were evaluated using a computer-assisted image analysis program (MetaMorph 6.1; Universal Imaging Corp., West Chester, PA, USA), which set the upper and lower thresholds for the immunofluorescence intensity determined by the signal. The images were collected using the same region and the same field sizes within the same lamina to avoid variations in staining between the laminae. The same configuration was used to measure the cell areas in all the experimental groups. The measured areas were automatically transferred to Microsoft Excel software (Microsoft Campus, Redmond, WA, USA) for subsequent statistical analysis. MetaMorph 6.1 was calibrated to standardize area measurements. A standardized field area was sampled arbitrarily from regions within the randomly selected lesion-associated cortex sections.
Table I. Neurological deficit scores $24 \mathrm{~h}$ after reperfusion from $120 \mathrm{~min}$ of middle cerebral artery occlusion in the rat.

\begin{tabular}{|c|c|c|c|c|c|c|c|}
\hline \multirow[b]{2}{*}{ Group } & \multicolumn{6}{|c|}{ Neurological deficit score } & \multirow{2}{*}{$\begin{array}{c}\text { Median } \\
\text { (range) }\end{array}$} \\
\hline & 0 & 1 & 2 & 3 & 4 & 5 & \\
\hline Naive & 9 & 3 & & & & & $0(0-1)$ \\
\hline Sham- $\mathrm{O}_{2}$ & 8 & 4 & & & & & $0(0-1)$ \\
\hline Sham-ISO & 8 & 4 & & & & & $0(0-1)$ \\
\hline MCAO-O ${ }_{2}$ & & & 2 & 5 & 5 & & $3(2-4)$ \\
\hline MCAO-ISO & & 5 & 7 & & & & $2(1-2)$ \\
\hline
\end{tabular}

ISO, isoflurane; MCAO, middle cerebral artery occlusion.

The number of TLR4-positive cells within the same areas was counted. The GFAP and OX-42 immunoreactivities and the total number of TLR4-immunopositive cells within the superficial cortex were averaged across the five spinal sections for each experimental group. The number of cells double-labeled with TLR4 and GFAP or OX-42 in the superficial cortex were counted and the proportion of double-labeled GFAP or OX-42 cells compared with the total TLR4-positive cells was calculated. The data from the western blot analysis are expressed as the mean \pm standard deviation. A repeated-measures analysis of variance with the Bonferroni confidence interval adjustment was conducted. $\mathrm{P}<0.05$ was considered to indicate a statistically significant difference.

\section{Results}

ISO pretreatment attenuates neurological deficits, brain edema and cerebral infarct size following ischemia/reperfusion. The neurological deficit scores of the MCAO-ISO group at $24 \mathrm{~h}$ after reperfusion were lower than those of the $\mathrm{MCAO}-\mathrm{O}_{2}$ groups (Table I). No changes were observed in the sham- $\mathrm{O}_{2}$, sham-ISO and naive groups. The infarct volume of the MCAO-ISO group was significantly reduced compared with that of the $\mathrm{MCAO}-\mathrm{O}_{2}$ group $24 \mathrm{~h}$ after reperfusion $(9.12 \pm 0.07$ vs. $16.23 \pm 0.05 \% ; \mathrm{P}<0.05)$. The sham- $\mathrm{O}_{2}$, sham-ISO and naive groups had low infarct volumes. The prevention of brain edema is critical for the preservation of neurological function and survival following focal cerebral ischemia/reperfusion. The brain water content was examined in cerebral tissue from the left hemisphere $24 \mathrm{~h}$ after cerebral ischemia/reperfusion. As shown in Fig. 1C, the brain water content was significantly increased in the MCAO- $\mathrm{O}_{2}$ group compared with the sham- $\mathrm{O}_{2}$ group $(87.29 \pm 0.09$ vs. $79.60 \pm 0.17 \%)$. However, the brain water content in the MCAO-ISO group was significantly lower than that in the MCAO-O 2 group $(82.61 \pm 0.08$ vs. $87.29 \pm 0.09 \%$; $\mathrm{P}<0.05)$. No significant difference in brain water content was detected among the sham- $\mathrm{O}_{2}$, sham-ISO and naive groups (Fig. 1).

ISO pretreatment reduces the protein expression of TLR4 and significantly attenuates astrocytic and microglial levels. Tissue sections of the right cortex were observed and the cerebral histological alterations caused by MCAO in rats were assessed using 
A

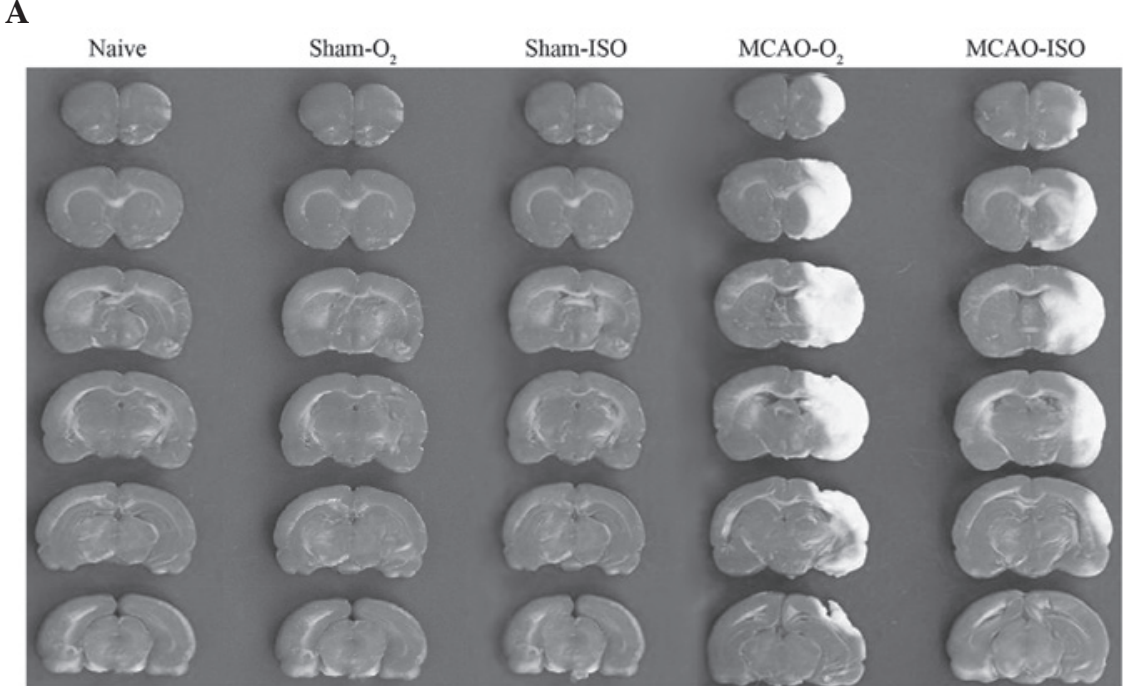

B

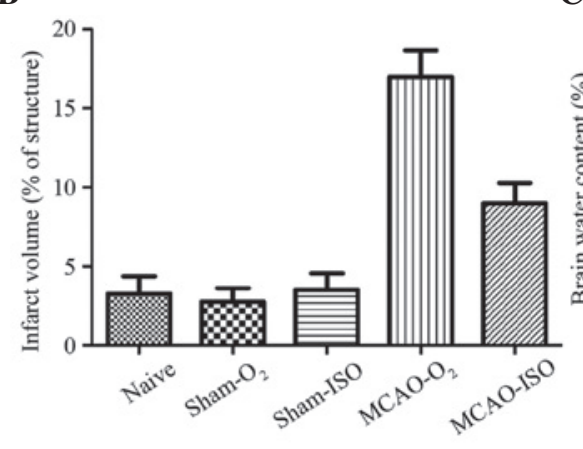

C

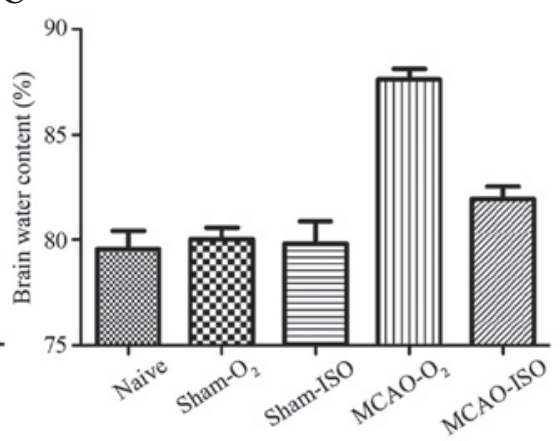

Figure 1. (A) Relative infarct volume using the triphenyltetrazolium chloride stain: A comparison of infarct size among the naive, sham- $\mathrm{O}_{2}$, sham-ISO, MCAO- $\mathrm{O}_{2}$ and MCAO-ISO groups at $24 \mathrm{~h}$. The white region indicates the infarction and the gray region indicates normal brain tissue. (B) Relative infarct volume was calculated by comparing the percentage of infarct region occupying the whole brain tissue area in one section. Data revealed that the infarct volume significantly decreased in the MCAO-ISO pretreatment group compared with the $\mathrm{MCAO}_{2} \mathrm{O}_{2}$ group at $24 \mathrm{~h}(\mathrm{P}<0.05)$. No difference was detected between naive, sham- $\mathrm{O}_{2}$ and sham-ISO. (C) Water content of cerebral tissue. Water content in the MCAO-ISO pretreatment group significantly decreased following surgery compared with the $\mathrm{MCAO}-\mathrm{O}_{2}$ group $(\mathrm{P}<0.05)$. No difference was detected in the naive, sham- $\mathrm{O}_{2}$ and sham-ISO groups. MCAO, middle cerebral artery occlusion; ISO, isoflurane.

a confocal laser-scanning microscope. Immunofluorescence histochemistry was employed to demonstrate TLR4 expression in the different groups. In order to rule out possible grouping and treatment bias, the number of TLR4-IR cells was normalized to that in naive rats $24 \mathrm{~h}$ after MCAO. MCAO significantly increased TLR4 expression in the cerebral cortex, which predominantly concentrated in the lesion-associated cortex. However, ISO pretreatment significantly inhibited MCAO-induced TLR4 protein expression in the cerebral cortex. Statistical analysis revealed that ISO pretreatment significantly affected the MCAO-induced cerebral cortex TLR4 expression. The TLR4 immunodensity in the MCAO-ISO rats was notably decreased by ISO pretreatment compared with that in the MCAO- $\mathrm{O}_{2}$ group. No alterations were observed in the sham- $\mathrm{O}_{2}$, sham-ISO and naive groups (Fig. 2).

Similarly to previous observations (22), the present results demonstrated that MCAO significantly induced GFAP and OX-42 expression in the lesion-associated cortex. Immunohistochemistry indicated that the activated astrocytes and microglia exhibited hypertrophied cell bodies and thickened processes with enhanced GFAP immunoreactivity and OX-42 immunoreactivity. Double immunofluorescence labeling indicated that multiple GFAP-IR cells were positive for TLR4, whereas fewer OX-42-IR cells were interspersed with activated microglia (Fig. 3). The activated astrocytes and microglia were predominantly distributed in the superficial cortex.

ISO pretreatment affected MCAO-induced TLR4 protein expression in the cerebral cortex. The immunodensities of GFAP and OX-42 in the MCAO rats were notably decreased by ISO pretreatment compared with those in the sham- $\mathrm{O}_{2}$ and sham-ISO groups (Fig. 4).

The data suggested that TLR4 is predominantly expressed in the astrocytes and microglia within the lesion-associated region. ISO pretreatment also suppressed MCAO-induced GFAP and OX-42 expression and inhibited astrocytic and microglial activation.

ISO pretreatment attenuates the protein expression levels

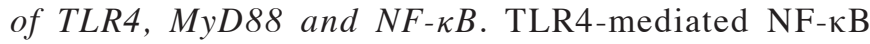
signaling contributes to myocardial ischemia-reperfusion injury (23). Quantitative studies using western blot analysis were performed to further observe whether ISO pretreatment affected the expression levels of TLR4, MyD88 and NF-кB. 


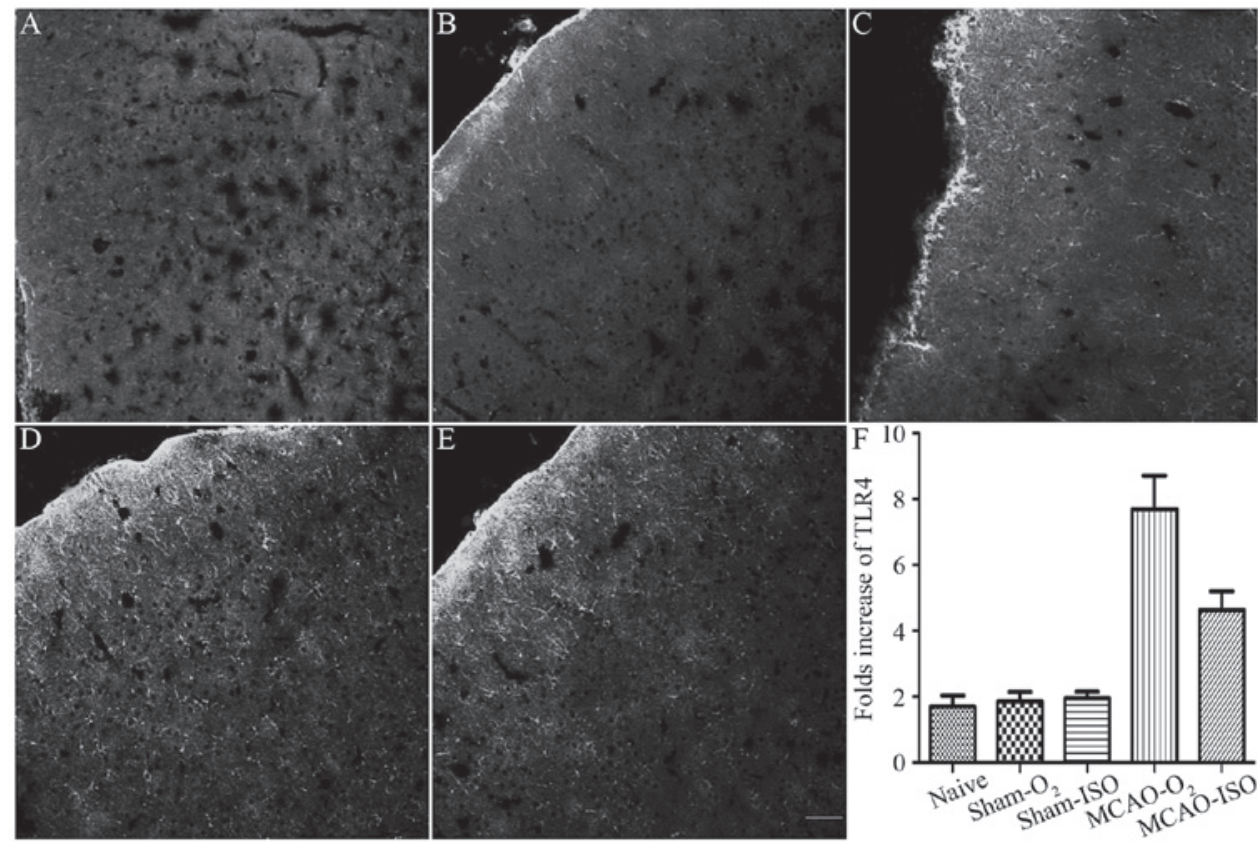

Figure 2. (A-C) Limited levels of TLR4 expression were detected in the naive, sham- $\mathrm{O}_{2}$ and sham-ISO groups. (D) MCAO induced significant TLR4 expression, (E) which may be suppressed by ISO pretreatment. (F) TLR4 positive cells observed in the in the cerebral cortex were counted, representing the expression of TLR4 following different treatments. TLR4, toll-like receptor 4; ISO, isoflurane; MCAO, middle cerebral artery occlusion.

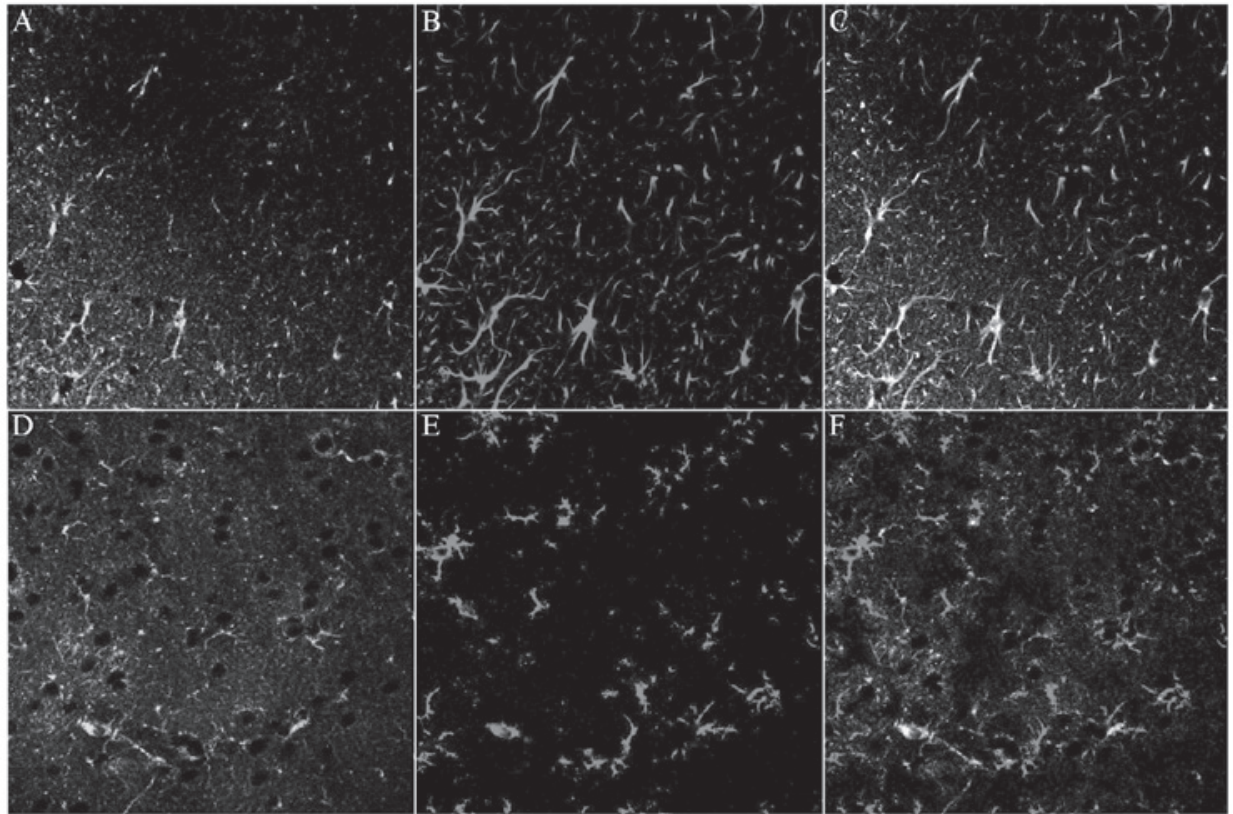

Figure 3. (A) Double immunofluorescence for TLR4 (green)/ (B) GFAP (red) and (D) TLR4 (green)/ (E) OX-42 (red) in the lesion-associated cortex following middle cerebral artery occlusion, respectively. Double immunofluorescence indicated that few OX-42-immunoreactive microglia were (F) TLR4 positive while the majority were $(\mathrm{C})$ nuclei of activated astrocytes. These activated microglia and astrocytes were predominantly distributed in the lesion-associated cortex and TLR4-positive astrocytes were surrounded with multiple GFAP-positive astrocytic processes forming close associations with each other. TLR4, toll-like receptor 4; GFAP, glial fibrillary acidic protein.

The protein expression levels of TLR4, MyD88 and $\mathrm{NF}-\mathrm{kB}$ were examined in the right cortex using western blot analysis at 24, 48 and $72 \mathrm{~h}$. As shown in Fig. 5, ISO pretreatment significantly inhibited MCAO-induced TLR4 expression compared with that in the $\mathrm{MCAO}-\mathrm{O}_{2}$ group. The protein level of TLR4 peaked at $24 \mathrm{~h}$ and lasted up to $48 \mathrm{~h}$. Thereafter, the protein level of TLR4 gradually decreased until $72 \mathrm{~h}$, although it remained significantly higher than those in the sham- $\mathrm{O}_{2}$, sham-ISO and naive groups. No significant difference in TLR4 expression was observed among the sham- $\mathrm{O}_{2}$, sham-ISO and naive groups. Furthermore, in accordance with the immunohistochemical results, the differences between the MCAO- $\mathrm{O}_{2}$ and MCAO-ISO groups were significant $(\mathrm{P}<0.05$; Fig. 4).

Similar to the effect of ISO pretreatment on Myd88 and $\mathrm{NF}-\kappa \mathrm{B}$ expression in the MCAO model, ISO pretreatment 


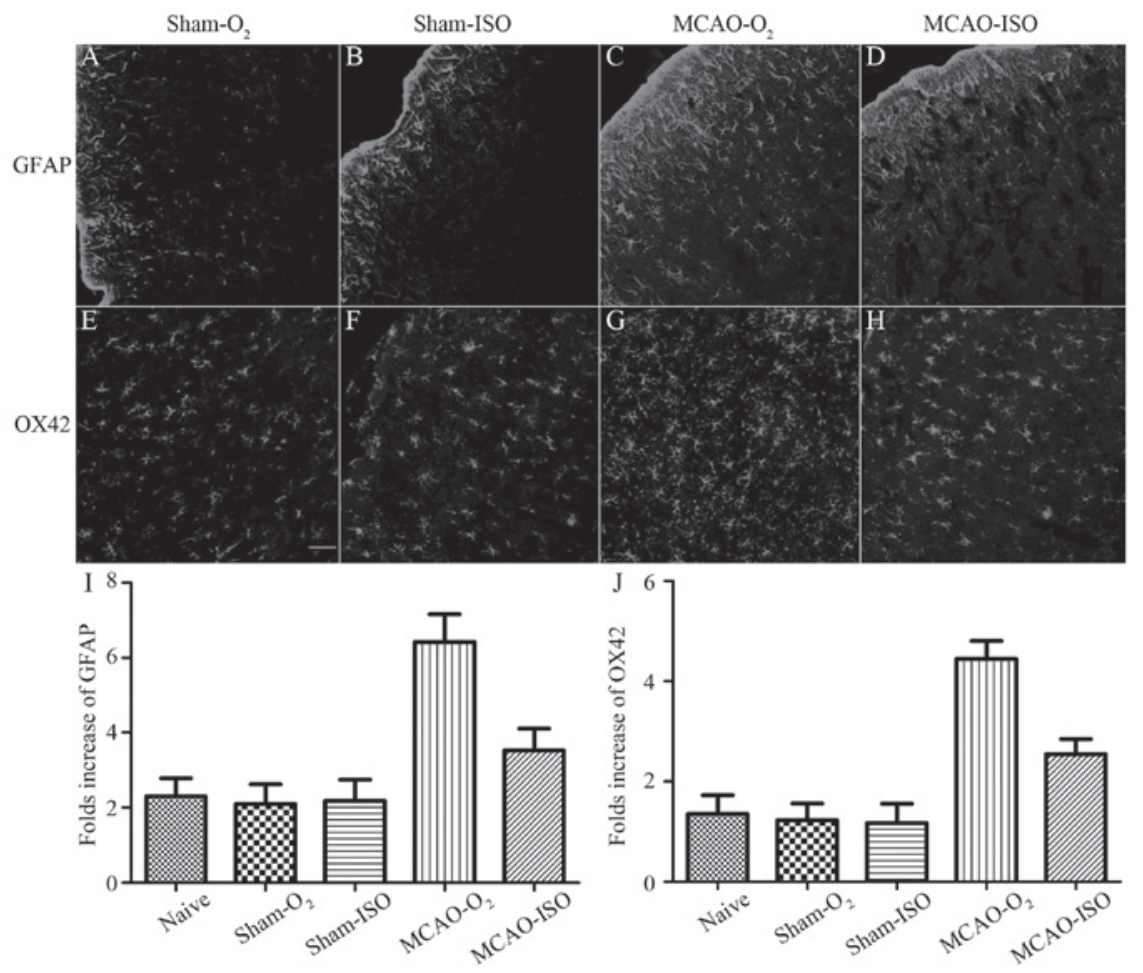

Figure 4. Expression levels of (A-D) GFAP and (E-H) OX-42 in the lesion-associated cortex in sham- $\mathrm{O}_{2}$, sham-ISO, MCAO- ${ }_{2}$ and MCAO-ISO groups, respectively, following MCAO. Compared with the $\mathrm{MCAO}-\mathrm{O}_{2}$-treated group, MCAO-ISO produced a significant inhibitory effect on GFAP and OX-42 protein expression, as well as on astrocyte and microglia inhibition in the cerebral cortex. GFAP and OX-42 labeled astrocytes and microglia observed in the cerebral cortex were counted. (I and J) Statistical results of these comparisons. TLR4, toll-like receptor 4; GFAP, glial fibrillary acidic protein; MCAO, middle cerebral artery occlusion; ISO, isoflurane.

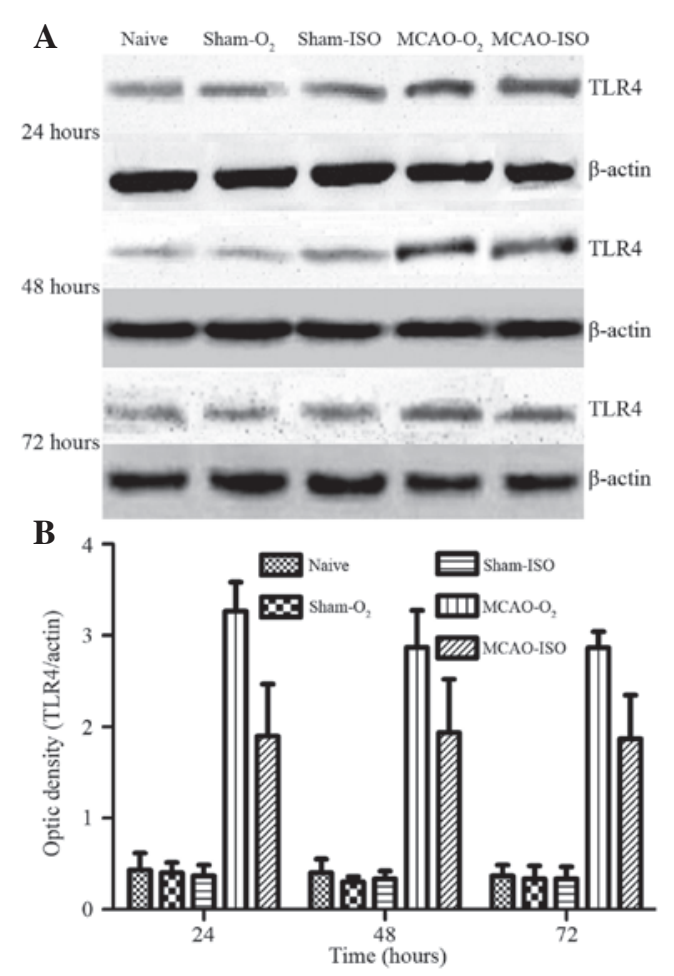

Figure 5. Western blot quantification of the expression levels of TLR4, myeloid differentiation primary response 88 and nuclear factor- $\mathrm{\kappa} B$ following different treatments. (A) Western blotting of TLR4 at 24, 48 and $72 \mathrm{~h}$, respectively, in brain tissue following surgery in each group. (B) Analysis of TLR4 revealed that ISO pretreatment significantly inhibited MCAO-induced TLR4 upregulation in the $\mathrm{MCAO}-\mathrm{O}_{2}$ group. ${ }^{*} \mathrm{P}<0.05$ vs. the MCAO-ISO group; ${ }^{* *} \mathrm{P}<0.01$ vs. the sham- $\mathrm{O}_{2}$ or sham-ISO group. TLR4, toll-like receptor 4; $\mathrm{MCAO}$, middle cerebral artery occlusion; ISO, isoflurane.

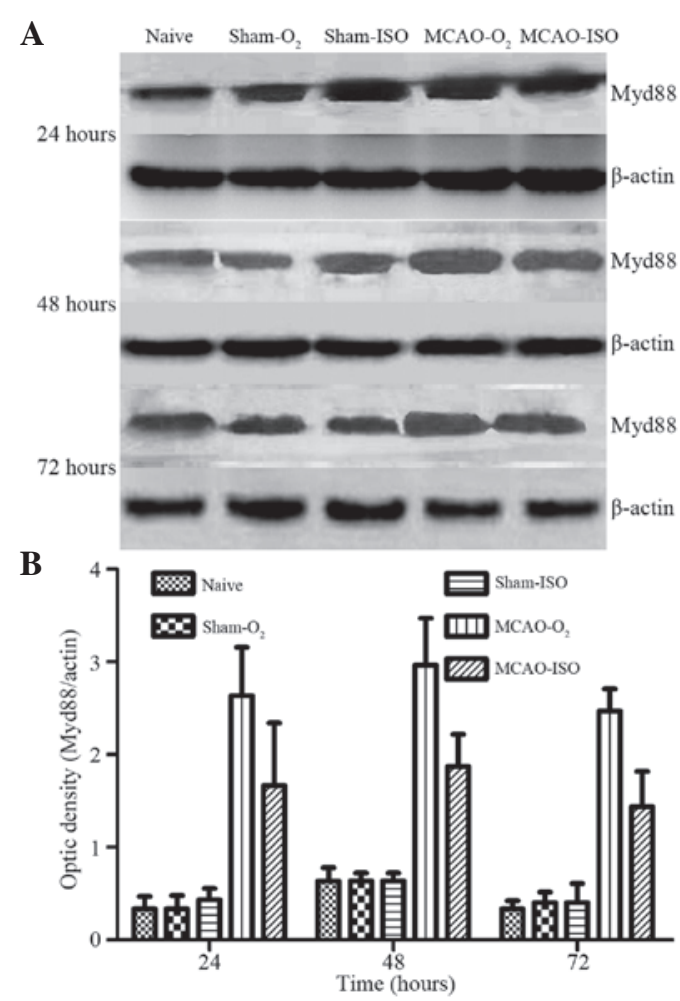

Figure 6. (A) Western blotting of Myd88 at 24, 48 and $72 \mathrm{~h}$, respectively, in brain tissue following surgery in each group. (B) Compared with the naive group, no difference was detected in Myd88 expression in the sham- $\mathrm{O}_{2}$ or sham-ISO group. However, ISO pretrement downregulated MCAO-induced Myd88 expression in the MCAO-ISO group. ${ }^{*} \mathrm{P}<0.05 .(\mathrm{n}=6)$, MCAO-ISO compared with the MCAO- $\mathrm{O}_{2}$. Myd88, myeloid differentiation primary response 88; MCAO, middle cerebral artery occlusion; ISO, isoflurane. 


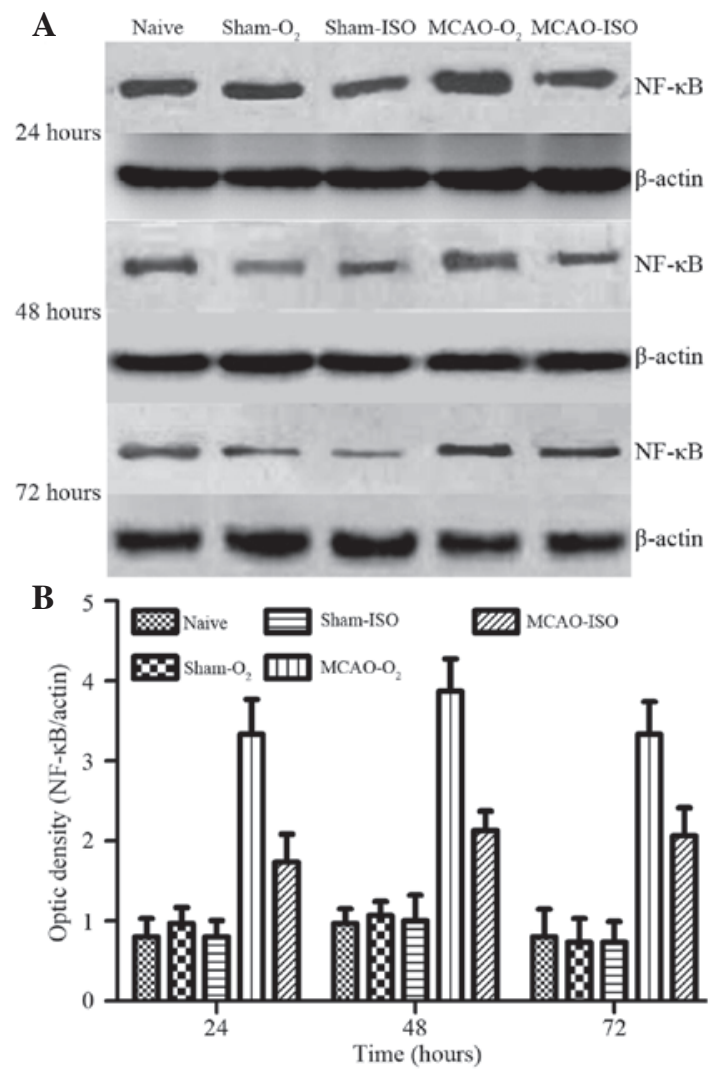

Figure 7. (A) Western blotting of NF- $\mathrm{KB}$ at 24, 48 and $72 \mathrm{~h}$, respectively, in brain tissue following surgery in each group.(B) Compared with the naive group, no difference was detected in NF- $\kappa \mathrm{B}$ expression in the sham- $\mathrm{O}_{2}$ or sham-ISO group. The protein expression of NF- $\mathrm{B}$ was significantly higher in the $\mathrm{MCAO}-\mathrm{O}_{2}$ group compared with that in the MCAO-ISO group $(\mathrm{P}<0.05)$. (B) Trend of the expression of MyD88 and $\mathrm{NF}-\kappa \mathrm{B}$ proteins was similar to that of the TLR4 protein. ISO, isoflurane; MCAO, middle cerebral artery occlusion; $\mathrm{NF}-\kappa \mathrm{B}$, nuclear factor- $\kappa \mathrm{B}$.

significantly downregulated Myd88 expression following MCAO compared with that in the $\mathrm{MCAO}-\mathrm{O}_{2}$ group $(\mathrm{P}<0.05)$. The protein level of Myd88 peaked at $48 \mathrm{~h}$ and then declined at $72 \mathrm{~h}$, although it remained significantly higher than those in the sham- $\mathrm{O}_{2}$, sham-ISO and naive groups. No significant difference in Myd88 expression was observed among the sham- $\mathrm{O}_{2}$, sham-ISO and naive groups (Fig. 6). ISO pretreatment significantly inhibited MCAO-induced NF- $\mathrm{B}$ expression compared with that in the MCAO- $\mathrm{O}_{2}$ group $(\mathrm{P}<0.05)$. The protein level of $\mathrm{NF}-\kappa \mathrm{B}$ peaked at $48 \mathrm{~h}$ and then declined at $72 \mathrm{~h}$, although it remained significantly higher than those in the sham- $\mathrm{O}_{2}$, sham-ISO and naive groups. Furthermore, no significant difference in $\mathrm{NF}-\kappa \mathrm{B}$ expression was observed among the sham- $\mathrm{O}_{2}$, sham-ISO and naive groups (Fig. 7).

\section{Discussion}

In the present study, the effects of ISO pretreatment on MCAO-induced TLR4, Myd88 and NF- $\kappa \mathrm{B}$ expression were examined, as well as on astrocytic and microglial activation. The present findings established the following: i) ISO pretreatment attenuated the neurological deficits, brain edema and cerebral infarct volume caused by ischemia/reperfusion; ii) ISO pretreatment potently inhibits TLR4 expression and significantly inhibited astrocytic and microglial activation in brain tissues and iii) ISO pretreatment downregulates the protein expression of TLR4, MyD88 and NF- $\mathrm{NB}$.

The anti-inflammatory effects of ISO during cerebral ischemia injury have attracted attention in previous years. Repeated $1 \mathrm{~h}$ ISO treatment induces a dose-dependent neuroprotection against subsequent ischemic injury (24). Additionally, ISO pretreatment reduces the infarct size after a 48 h delay; however, the protective effect of ISO pretreatment may decrease after $72 \mathrm{~h}$ (25). In the present study, the potential involvement of TLR4 in the anti-inflammatory activity elicited by ISO pretreatment was examined. The present study revealed that pretreatment with $2 \%$ ISO ( $1 \mathrm{~h} /$ day) for 5 days provided significant neuroprotection against the neurological injury induced by MCAO. ISO pretreatment also reduced the water content and volume of the infarct in the ischemic brain tissue.

Cerebral infarction is a complex pathophysiological process, which involves multiple factors (26). Injured tissue releases endogenous molecules, which in turn induce an inflammatory immune response. A number of these endogenous components are ligands for TLR4 (27). TLR4 has important functions in cerebral ischemia and in the induction of immunoinflammatory reactions (28). TLR4, as a key component of the innate immune system, functions as a pattern recognition receptor that recognizes lipopolysaccharide (LPS). LPS is one of the most immunostimulatory glycolipid components of the outer membrane of Gram-negative bacteria (29). The TLR4-mediated MyD88-dependent signaling pathway is essential for $\mathrm{NF}-\kappa \mathrm{B}$ activation (30). MyD88 recruitment to the toll-interleukin receptor domain of TLR4 activates $\mathrm{NF}-\kappa \mathrm{B}$, which is involved in cerebral ischemia/reperfusion injury $(31,32)$.

In the present study, MCAO significantly increased TLR4 expression in the cerebral cortex and was more concentrated in the lesion-associated cortex. However, TLR4 declined following ISO pretreatment. In addition, ISO pretreatment potently inhibited TLR4 expression, which was correlated with a decrease in astrocytic and microglial activation. In order to understand the mechanisms involved in regulating the inflammatory response during cerebral ischemia, TLR4 colocalization in astrocytes and microglial cells was investigated. Astrocytes and microglial cells are involved in the local innate immune response triggered by various stressors $(33,22)$. Certain functions of astrocytes and microglia are ambiguous (34). When activated, microglial cells rapidly migrate to the sites of brain damage and clear debris to maintain the integrity of the central nervous system $(35,36)$. Astrocytes secrete soluble mediators, which affect the innate and adaptive immune responses $(37,15)$. The two cell types produce neuroprotective growth factors and neurotrophins, which repair brain tissue. However, microglial cells can cause severe neuronal damage when pro-inflammatory immune mediators are produced excessively (35). By contrast, astrocytes promote inflammation through NF- $\kappa \mathrm{B}$-dependent pathways $(38,39)$. In the present study, immunohistochemical staining demonstrated that activated astrocytes and microglia in the $\mathrm{MCAO}-\mathrm{O}_{2}$ group exhibited hypertrophied cell bodies and thickened processes with enhanced GFAP immunoreactivity and OX-42 immunereactivity. Double immunofluorescence labeling indicated that several of the GFAP-IR cells were positive for TLR4, whereas a few OX-42-IR cells overlapped with TLR4. Notably, it was 
observed that the immunodensity of GFAP and OX-42 in the MCAO rats significantly decreased following ISO pretreatment compared with those in the sham- $\mathrm{O}_{2}$ and sham-ISO groups.

The protein levels of TLR4, Myd88 and NF-KB in each group were determined using western blot analysis to determine whether or not ISO pretreatment protects rat brains against focal ischemia through the TLR4-mediated MyD88-dependent signaling pathway. The protein level of TLR4 in the MCAO- $\mathrm{O}_{2}$ group was significantly higher than that in the MCAO-ISO group. The expression level of TLR4 in the ischemic brains peaked at $24 \mathrm{~h}$, lasted until $48 \mathrm{~h}$ and then gradually decreased at $72 \mathrm{~h}$. These findings suggested that the inflammatory peak occurred after $24 \mathrm{~h}$ of ischemia. The expression level of MyD88 in the ischemic brains increased at $48 \mathrm{~h}$ but decreased at $72 \mathrm{~h}$. In addition, the expression level of NF- $\mathrm{BB}$ increased at $48 \mathrm{~h}$ but decreased $72 \mathrm{~h}$ after MCAO. These results suggested that the level of TLR4 in the right cortex is attenuated by ISO pretreatment during cerebral ischemia. The pattern of MyD88 and NF- $\mathrm{kB}$ expression was similar to that of TLR4 expression.

The protein expression levels of MyD88 and NF- $\kappa \mathrm{B}$ increased later than that of the TLR4 protein. This result can be attributed to the involvement of MyD88 and NF- $\mathrm{kB}$ in the TLR4-mediated signaling pathway activated by cerebral ischemia.

In conclusion, the present study confirmed that ISO pretreatment protected the brain from the damage caused by MCAO. The neuroprotective effect of ISO pretreatment may be associated with downregulation of TLR4, MyD88 and NF- $\kappa B$ expression. Inflammatory responses mediated mainly by the activation of the TLR4-MyD88 signaling pathway may have an important function in the pathogenesis of cerebral ischemia. The effect of ISO pretreatment on the TLR4-MyD88 signaling pathway is important during the acute phase of ischemia and may provide new perspectives for therapeutic targets in patients with cerebral ischemia.

\section{Acknowledgements}

This study was supported by the National Natural Science Foundation of China (grant no. 81271343) and by the Shaanxi Science and Technology research fund (grant no. 2014K11-02-01-14).

\section{References}

1. Fan H and Cook JA: Molecular mechanisms of endotoxin tolerance. J Endotoxin Res 10: 71-84, 2004.

2. Aderem A and Ulevitch RJ: Toll-like receptors in the induction of the innate immune response. Nature 406: 782-787, 2000.

3. Chakravarty S and Herkenham M: Toll-like receptor 4 on nonhematopoietic cells sustains CNS inflammation during endotoxemia, independent of systemic cytokines. J Neurosci 25: 1788-1796, 2005.

4. Kaisho T and Akira S: Toll-like receptors as adjuvant receptors. Biochim Biophys Acta 1589: 1-13, 2002.

5. Kilic U, Kilic E, Matter CM, Bassetti CL and Hermann DM TLR-4 deficiency protects against focal cerebral ischemia and axotomy-induced neurodegeneration. Neurobiol Dis 31: 33-40, 2008.

6. Kitano H, Young JM, Cheng J, Wang L, Hurn PD and Murphy SJ: Gender-specific response to isoflurane preconditioning in focal cerebral ischemia. J Cereb Blood Flow Metab 27: 1377-1386, 2007.
7. Zhang J, Yang ZJ, Klaus JA, Koehler RC and Huang J: Delayed tolerance with repetitive transient focal ischemic preconditioning in the mouse. Stroke 39: 967-974, 2008.

8. Eilers H and Bickler PE: Hypothermia and isoflurane similarly inhibit glutamate release evoked by chemical anoxia in rat cortical brain slices. Anesthesiology 85: 600-607, 1996.

9. Becker K, Eder M, Ranft A, et al: Low dose isoflurane exerts opposing effects on neuronal network excitability in neocortex and hippocampus. PLoS One 7: e39346, 2012.

10. Puil E, El-Beheiry H and Baimbridge KG: Anesthetic effects on glutamate-stimulated increase in intraneuronal calcium. J Pharmacol Exp Ther 255: 955-961, 1990.

11. Puil E and El-Beheiry H: Anaesthetic suppression of transmitter actions in neocortex. Br J Pharmacol 101: 61-66, 1990.

12. Bickler PE, Buck LT and Hansen BM: Effects of isoflurane and hypothermia on glutamate receptor-mediated calcium influx in brain slices. Anesthesiology 81: 1461-1469, 1994.

13. Dong Y and Benveniste EN: Immune function of astrocytes. Glia 36: 180-190, 2001.

14. Ladeby R, Wirenfeldt M, Garcia-Ovejero D, et al: Microglial cell population dynamics in the injured adult central nervous system. Brain Res Brain Res Rev 48: 196-206, 2005.

15. Song JH, Bellail A, Tse MC, Yong VW and Hao C: Human astrocytes are resistant to Fas ligand and tumor necrosis factor-related apoptosis-inducing ligand-induced apoptosis. J Neurosci 26: 3299-3308, 2006.

16. Li H, Yin J, Li L, Deng J, Feng C and Zuo Z: Isoflurane postconditioning reduces ischemia-induced nuclear factor-kappaB activation and interleukin 1beta production to provide neuroprotection in rats and mice. Neurobiol Dis 54: 216-224, 2013.

17. Bedirli N, Bagriacik EU, Emmez H, Yilmaz G, Unal Y and Ozkose Z: Sevoflurane and isoflurane preconditioning provides neuroprotection by inhibition of apoptosis-related mRNA expression in a rat model of focal cerebral ischemia. J Neurosurg Anesthesiol 24: 336-344, 2012.

18. Chen J, Graham SH, Zhu RL and Simon RP: Stress proteins and tolerance to focal cerebral ischemia. J Cereb Blood Flow Metab 16: 566-577, 1996.

19. Longa EZ, Weinstein PR, Carlson S and Cummins R: Reversible middle cerebral artery occlusion without craniectomy in rats. Stroke 20: 84-91, 1989.

20. Shimamura N, Matchett G, Solaroglu I, Tsubokawa T, Ohkuma $\mathrm{H}$ and Zhang J: Inhibition of integrin alphavbeta3 reduces blood-brain barrier breakdown in focal ischemia in rats. J Neurosci Res 84: 1837-1847, 2006.

21. Guo W, Wang H, Watanabe M, et al: Glial-cytokine-neuronal interactions underlying the mechanisms of persistent pain. J Neurosci 27: 6006-6018, 2007.

22. Tang SC, Arumugam TV, Xu X, et al: Pivotal role for neuronal Toll-like receptors in ischemic brain injury and functional deficits. Proc Natl Acad Sci USA 104: 13798-13803, 2007.

23. Hua F, Ma J, Ha T, et al: Activation of Toll-like receptor 4 signaling contributes to hippocampal neuronal death following global cerebral ischemia/reperfusion. J Neuroimmunol 190: 101-111, 2007.

24. Xiong L, Zheng Y, Wu M, et al: Preconditioning with isoflurane produces dose-dependent neuroprotection via activation of adenosine triphosphate-regulated potassium channels after focal cerebral ischemia in rats. Anesth Analg 96: 233-237, 2003.

25. Kawaguchi M, Drummond JC, Cole DJ, Kelly PJ, Spurlock MP and Patel PM: Effect of isoflurane on neuronal apoptosis in rats subjected to focal cerebral ischemia. Anesth Analg 98: 798-805, 2004.

26. Hanisch UK and Kettenmann H: Microglia: active sensor and versatile effector cells in the normal and pathologic brain. Nat Neurosci 10: 1387-1394, 2007.

27. Kapinya KJ, Lowl D, Futterer C, et al: Tolerance against ischemic neuronal injury can be induced by volatile anesthetics and is inducible NO synthase dependent. Stroke 33: 1889-1898, 2002.

28. Lee SJ and Lee S: Toll-like receptors and inflammation in the CNS. Curr Drug Targets Inflamm Allergy 1: 181-191, 2002.

29. Beg AA: Endogenous ligands of Toll-like receptors: implications for regulating inflammatory and immune responses. Trends Immunol 23: 509-512, 2002.

30. Doyle SL and O'Neill LA: Toll-like receptors: from the discovery of NFkappaB to new insights into transcriptional regulations in innate immunity. Biochem Pharmacol 72: 1102-1113, 2006.

31. Baeuerle PA and Henkel T: Function and activation of NF-kappaB in the immune system. Annu Rev Immunol 12: 141-179, 1994. 
32. Seki E, Tsutsui H, Iimuro Y, et al: Contribution of Toll-like receptor/myeloid differentiation factor 88 signaling to murine liver regeneration. Hepatology 41: 443-450, 2005.

33. Farina C, Aloisi F and Meinl E: Astrocytes are active players in cerebral innate immunity. Trends Immunol 28: 138-145, 2007.

34. Glezer I, Simard AR and Rivest S: Neuroprotective role of the innate immune system by microglia. Neuroscience 147: 867-883, 2007.

35. Aloisi F: Immune function of microglia. Glia 36: 165-179, 2001.

36. Streit WJ, Conde JR, Fendrick SE, Flanary BE and Mariani CL: Role of microglia in the central nervous system's immune response. Neurol Res 27: 685-691, 2005.
37. Akira S, Uematsu S and Takeuchi O: Pathogen recognition and innate immunity. Cell 124: 783-801, 2006.

38. Bowman CC, Rasley A, Tranguch SL and Marriott I: Cultured astrocytes express toll-like receptors for bacterial products. Glia 43: 281-291, 2003.

39. Hang CH, Shi JX, Li JS, Wu W and Yin HX: Concomitant upregulation of nuclear factor- $\kappa \mathrm{B}$ activity, proinflammatory cytokines and ICAM-1 in the injured brain after cortical contusion trauma in a rat model. Neurol India 53: 312-317, 2005. 